\title{
Rhein shows potent efficacy against non-small-cell lung cancer through inhibiting the STAT3 pathway
}

This article was published in the following Dove Medical Press journal:

Cancer Management and Research

\section{Lehe Yang, ${ }^{1-3, *}$ Jifa Li, ${ }^{1}, *$ Lingyuan $\mathrm{Xu},{ }^{1-3, *}$ Shichong Lin, ${ }^{1-3}$ Youqun Xiang, ${ }^{3}$ Xuanxuan Dai, ${ }^{3}$ Guang Liang, ${ }^{2}$ Xiaoying Huang, ${ }^{3}$ Jiandong Zhu,' Chengguang Zhao ${ }^{1,2}$}

'Department of Respiratory Medicine, Affiliated Yueqing Hospital, Wenzhou Medical University, Wenzhou, Zhejiang 325600, People's Republic of China; ${ }^{2}$ Chemical Biology Research Center, School of Pharmaceutical Sciences, Wenzhou Medical University, Wenzhou, Zhejiang 325035, People's Republic of China; ${ }^{3}$ Division of Pulmonary Medicine, The First Affiliated Hospital of Wenzhou Medical University, Key Laboratory of Heart and Lung, Wenzhou, Zhejiang 325000, People's Republic of China

*These authors contributed equally to this work
Correspondence: Chengguang Zhao Chemical Biology Research Center, School of Pharmaceutical Sciences, Wenzhou Medical University, Building II, Chashan Street, University Town, Wenzhou, Zhejiang 325035, People's Republic of China

Tel/fax +86 57786699057

Email zhaochengguang@wmu.edu.cn

Jiandong Zhu

Department of Respiratory Medicine, Affiliated Yueqing Hospital, Wenzhou Medical University, Wenzhou, Zhejiang 325600, People's Republic of China Email 873331666@qq.com
Background: Non-small-cell lung cancer (NSCLC) comprises about $85 \%$ of all lung cancers and is usually diagnosed at an advanced stage with poor prognosis. The IL-6/STAT3 signaling pathway plays a pivotal role in NSCLC biology. Rhein is a lipophilic anthraquinone extensively found in medicinal herbs. Emerging evidence suggests that Rhein has significant antitumor effects, supporting the potential uses of Rhein as an antitumor agent.

Methods: Cell viability and colony formation were performed to examine Rhein's potent antiproliferative effect in human NSCLC cell lines PC-9, H460 and A549. Flow cytometry-based assay was employed to study whether Rhein could affect cell apoptosis and cycle. The expression level of P-STAT3, apoptosis and cycle-related proteins Bcl-2, Bax, MDM2, CDC2, P53 and CyclinB1 were detected by Western blotting. The xenograft models were used to evaluate the in vivo effect of Rhein.

Results: We found that Rhein could significantly reduce the viability and stimulate apoptosis in human NSCLC cells in a dose-dependent manner. Western blot analysis results suggested that the antitumor effect of Rhein might be mediated via STAT3 inhibition. Rhein upregulated the expression of the proapoptotic protein Bax and downregulated the expression of the antiapoptotic protein Bcl-2. In addition, Rhein induced the arrest of NSCLC cells in the G2/M phase of the cell cycle and dose dependently inhibited the expression of cycle-related proteins. The Rhein also inhibited tumor growth in H460 xenograft models.

Conclusion: Rhein shows potent efficacy against NSCLC through inhibiting the STAT3 pathway. Our results also suggest that Rhein has a promising potential to be used as a novel antitumor agent for the treatment of NSCLC.

Keywords: Rhein, NSCLC, STAT3, EGFR, diacerein, apoptosis, inhibitor

\section{Introduction}

Lung cancer is the leading cause of death from cancer worldwide and is responsible for nearly one in five cancer deaths. ${ }^{1}$ Only $17.7 \%$ of all patients with lung cancer can live $\geq 5$ years after diagnosis. ${ }^{2}$ Non-small-cell lung cancer (NSCLC) represents about $85 \%$ of all lung cancers. ${ }^{3}$ Up to $69 \%$ of the advanced NSCLC patients could have a potentially actionable molecular target. ${ }^{4}$ However, for patients with advanced NSCLC who do not fit an approved molecular targeted therapy, the standard first-line treatment remains platinum-based doublet therapy. Although targeted drugs against epidermal growth factor receptor (EGFR) have been increasingly developed for the treatment of NSCLC, unfortunately, nearly all patients eventually have disease progression due to acquired resistance. 
As an important member of the signal transducer and activator of transcription family (STAT), STAT3 is associated with malignant transformation and tumor progression. ${ }^{5,6} \mathrm{Con}$ stitutive activation of STAT3-meditated signal pathway plays pivotal roles in tumor cell growth, survival, apoptosis, angiogenesis and metastasis. ${ }^{7,8}$ Growing evidence demonstrates that constitutively activated STAT3 contributes to tumor development and progression in the majority of cancers, including breast, prostate, ovary, lung, gastric, pancreatic, melanoma and blood cancers. ${ }^{9-12}$ STAT3 is persistently activated in $22 \%-65 \%$ of NSCLC. ${ }^{13-15}$ Several studies suggest that the high expression of P-STAT3 is a strong predictor of poor prognosis in patients with NSCLC. Previous findings reported that the STAT3 pathway was associated with intrinsic resistance to chemotherapeutic agents in several malignancies. ${ }^{16,17}$ You et al showed that ionizing radiation induces phosphorylation of JAK2 and STAT3, and higher expression of STAT3 was found in the nucleus of radioresistant NSCLC cells. ${ }^{18}$

STAT3 is also involved in one of the EGFR downstream pathways. ${ }^{19}$ EGFR can directly phosphorylate STAT3, and activation of STAT3 has also been reported in NSCLC cell lines harboring activated EGFR mutations. ${ }^{14,20,21}$ Studies also showed that EGFR inhibitors acting on cancer cells can activate the IL-6/JAK/STAT3 signaling pathway, thereby leading to drug resistance..$^{22,23,24}$ Although the response rate to EGFR tyrosine kinase inhibitor (TKI) is $\sim 80 \%$ in EGFRmutant patients, progression-free survival is only about 1 year, as most patients eventually develop acquired resistance to the TKIs. ${ }^{24}$ Several reports found that inhibition of STAT3 suppressed the growth of cancer cells and enhanced the sensitivity to antitumor agents in multiple types of cancer. ${ }^{26,27}$ Therefore, STAT3 has been considered a potential target for NSCLC therapy.

Currently, studies have focused on the antitumor properties of natural products because of their confirmed pharmacological properties and few side effects. Rhein is a lipophilic anthraquinone extensively found in medicinal herbs Rheum palmatum L., Cassia tora L. and so on, which have been used medicinally for $>1,000$ years. ${ }^{25}$ Rhein has many pharmacological effects, including hepatoprotective, nephroprotective, anti-inflammatory, anticancer, antioxidant and antimicrobial activities. Although several studies have reported the mechanisms and pathways of the antitumor effect of Rhein, the direct molecular targets and specific mechanism remain unclear. ${ }^{25}$ Diacerein, which is known to be completely metabolized into Rhein by humans and animals, is clinically prescribed for the treatment of osteoarthritis. ${ }^{26}$ In this study, we focus on the specific molecular mechanism of action of Rhein and Diacerein that exert their antitumor effects by inhibiting STAT3.

\section{Materials and methods Cell culture}

Human NSCLC cell lines PC-9, H460 and A549 were obtained from Shanghai Institute of Biosciences and Cell Resources Center (Chinese Academy of Sciences, Shanghai, People's Republic of China). All the cells were cultured in Roswell Park Memorial Institute-1640 media (Thermo Fisher Scientific, Waltham, MA, USA) with 10\% FBS (Thermo Fisher Scientific). Cells were cultured in a humidified cell incubator with an atmosphere of $5 \% \mathrm{CO}_{2}$ at $37^{\circ} \mathrm{C}$.

\section{Antibodies and reagents}

The antibodies against P-STAT3, STAT3, Bax, GAPDH, P53 and Cyclin B1 were purchased from Cell Signal Technology (Danvers, MA, USA). The antibodies against Bcl-2, MDM2, CDC2, horseradish peroxidase (HRP)-conjugated donkey anti-rabbit IgG and HRP-conjugated goat anti-mouse IgG were purchased from Santa Cruz Biotechnology Inc. (Dallas, TX, USA). MTT and dimethyl sulfoxide (DMSO) were purchased from Sigma-Aldrich Co. (St Louis, MO, USA). The caspase-3 colorimetric assay kit was purchased from Abcam Co. (Cambridge, MA, USA). The Annexin V-fluorescein isothiocyanate (FITC) apoptosis Detection Kit I and propidium iodide (PI) were purchased from BD Pharmingen (Franklin Lakes, NJ, USA). The Dual-Luciferase Report Assay Kit was obtained from Promega Biotech Co., Ltd (Madison, WI, USA). Rhein and Diacerein were purchased from Aladdin Biochemical Technology Company (Shanghai, People's Republic of China). The compounds used in vitro were dissolved in DMSO. A Bradford protein assay kit, polyvinylidene fluoride (PVDF) membrane and enhanced chemiluminescence kit were obtained from Bio-Rad Laboratories Inc. (Hercules, CA, USA). A protease phosphatase inhibitor mixture was obtained from Applygen Technologies (Beijing, People's Republic of China). Acrylamide (30\%), Coomassie Brilliant Blue, tetramethylethylenediamine, Trisglycine, sodium dodecyl sulfate, prestained protein marker and nonfat dry milk were from Bio-Rad Laboratories.

\section{MTT cytotoxicity assay}

MTT assay was utilized to measure human NSCLC cells' cytotoxicity and viability. Cells $\left(5 \times 10^{3}\right.$ cells/well $)$ were plated in 96-well plates and allowed to attach overnight. After appropriate treatment, an MTT solution was added at $25 \mu \mathrm{L} /$ well 
and incubated for 4 hours at $37^{\circ} \mathrm{C}$. The formazan crystals were dissolved in $150 \mu \mathrm{L}$ DMSO and the OD was measured using a microplate reader at $490 \mathrm{~nm}$. The cell viability was calculated according to the following formula: viability = (average OD values of treatment wells/average OD values of vehicle control wells) $\times 100 \%$. Also, the $\mathrm{IC}_{50}$ values were determined by GraphPad Pro Prism 7.0.

\section{Colony formation assay}

Human NSCLC cells (1,000 cells/well) were seeded in sixwell plates at $37^{\circ} \mathrm{C}$ in $5 \% \mathrm{CO}_{2}$ atmosphere overnight. DMSO (control), Rhein $(30,60,100 \mu \mathrm{M})$ or Diacerein $(30,60,100$ $\mu \mathrm{M}$ ) was added to the cells for 7 days. The culture medium was replaced with fresh medium every 2 days to keep the cells growing for 7 days. Colonies were washed with PBS, fixed with 4\% paraformaldehyde at room temperature for 15 minutes, washed with purified water for 3 times and stained with crystal violet for 10 minutes. Each experiment was done in triplicate for three independent experiments.

\section{Western blot analysis}

Cancer cells were seeded in six-well plates at a density of 500,000 cells/well and then incubated overnight. DMSO (control), Rhein $(30,60,100 \mu \mathrm{M})$ or Diacerein $(30,60,100 \mu \mathrm{M})$ was added into the six-well plates. Treated cells were washed with PBS and harvested using ice-cold RIPA lysis buffer with $1 \%$ phenylmethanesulfonyl fluoride. Proteins were separated by $10 \%$ SDS-PAGE and transferred onto a PVDF membrane, and then the blots were blocked for $2 \mathrm{~h}$ at room temperature with fresh $5 \%$ nonfat milk. The blots were incubated with specific primary antibodies. After washing, the membranes were incubated with the relevant secondary antibodies; visualization of bands was by enhanced chemiluminescence.

\section{Cell apoptosis assay}

PC-9 and H460 cells were seeded into six-well plates and allowed to grow to $80 \%$ confluency in complete medium. Then the cells were treated with DMSO (control), Rhein $(30,60,100 \mu \mathrm{M})$ or Diacerein $(30,60,100 \mu \mathrm{M})$ for 48 hours to evaluate the effects of these compounds on apoptosis. Cells were collected, washed twice in ice-cold PBS and then resuspended in binding buffer according to the instructions of the apoptosis kit. The treated cells (as described above) were simultaneously incubated with fluorescein-labeled Annexin $\mathrm{V}$ and PI. Annexin V-binding buffer was then added to the mixture before fluorescence was measured on a FACSCalibur (BD Biosciences, Baltimore, MD, USA). Data were analyzed using Flowjo software.

\section{Cell cycle assay}

Cells were seeded into six-well plates for 24 hours and then treated with DMSO or Rhein $(30,60,100 \mu \mathrm{M})$ for 24 hours. Cells were then labeled with PI, and the cell cycle was analyzed on a FACSCalibur.

\section{STAT3 luciferase report assay}

The STAT3 luciferase reporter plasmid (pGLSTAT3-Luc) was used to detect STAT3 activation. The H460 cells were seeded in 24-well plates 24 hours before transfection. Then, the cells were co-transfected with pGLSTAT3-Luc and pRL-TK, a plasmid encoding Renilla luciferase, using Lipofectamine 3000 (Thermo Fisher Scientific) for 6 hours. Finally, the cells were treated with the indicated concentrations of Rhein for 24 hours. Luciferase activity was assessed by SpectraMax ID3 (Molecular Devices, San Jose, CA, USA). The inhibition of STAT3 activation by Rhein was calculated as the ratio between the value of firefly and Renilla luciferase activity. Experiments were performed in triplicate.

\section{In vivo antitumor study}

The Wenzhou Medical University Animal Policy and Welfare Committee approved all mouse experiments. The H460 cells $\left(5 \times 10^{6}\right.$ cells were mixed with an equal volume of PBS and Matrigel in $100 \mu \mathrm{L}$ ) were implanted on the hind flank of mice (athymic nude female mice, 5-6 weeks old). Once the tumor volumes reached $\sim 50 \mathrm{~mm}^{3}$, mice were divided into three experimental groups (six mice per group, no differences in mean body weights or tumor volumes between the groups). Tumor volume was measured as $\mathrm{V}=(\mathrm{L} \times \mathrm{W} \times \mathrm{W}) / 2$, in which $\mathrm{L}$ and $\mathrm{W}$ represent length and width of tumor, respectively. Animals were sacrificed on day 10, 4 hours after the last Rhein treatment. The tumors, heart, liver, kidney and lung were removed for use in the histology and Western blot analysis.

\section{Statistical analysis}

Data were expressed as mean $\pm \mathrm{SD}$ of three independent experiments. The statistical differences between different groups were analyzed by the Student's $t$-test or one-way ANOVA in GraphPad Pro7.0 (GraphPad Software, Inc., La Jolla, CA, USA). $P$-values $<0.05$ were considered indicative of significance.

\section{Results}

\section{The effect of Rhein on NSCLC cell proliferation by the MTT and colony formation assays}

To investigate the effect of Rhein and Diacerein (Figure 1A) on human NSCLC cell lines, cell viability was evaluated 
A<smiles>O=C(O)c1cc(O)c2c(c1)C(=O)c1cccc(O)c1C2=O</smiles>

Rhein<smiles>CC(=O)Oc1cccc2c1C(=O)c1c(OC(C)=O)cc(C(=O)O)cc1C2=O</smiles>

Diacerein
B

C
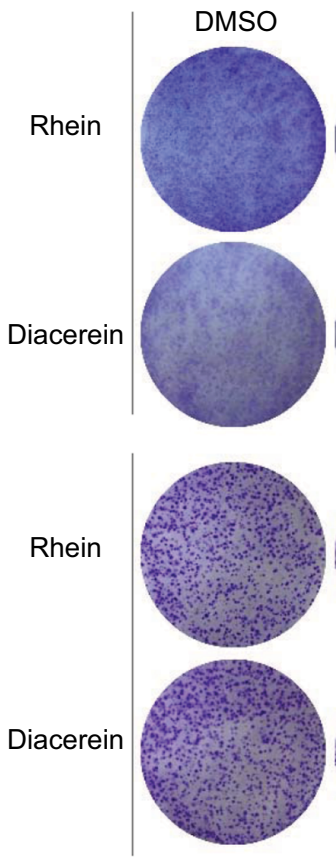

D

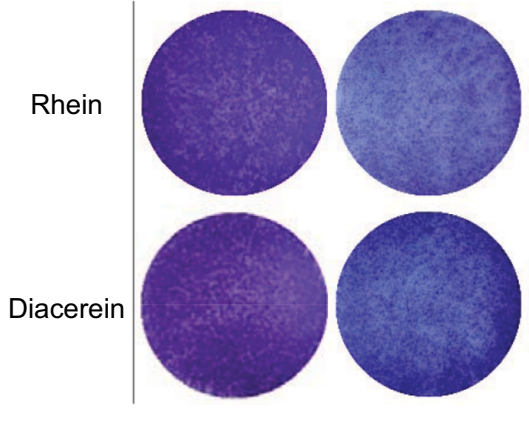

60
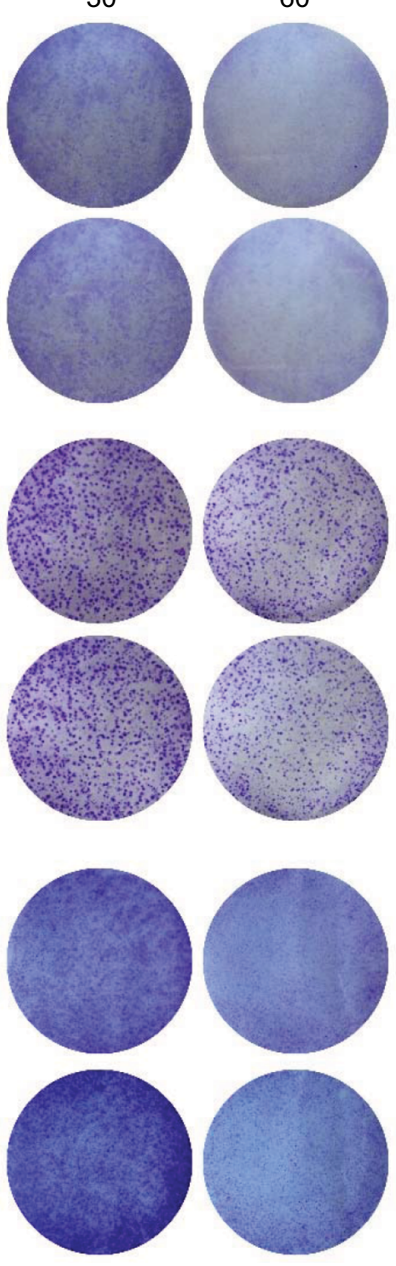

$100 \mu \mathrm{M}$
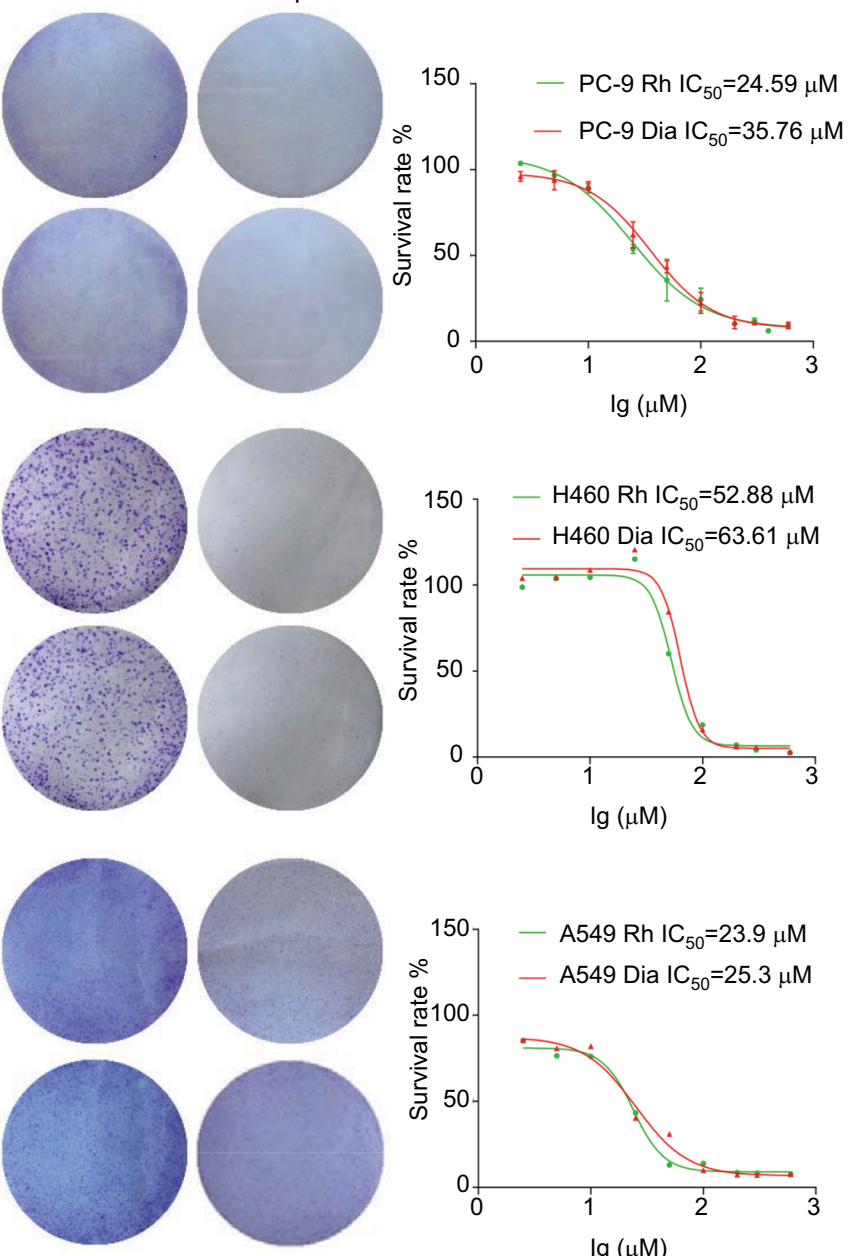

$\lg (\mu \mathrm{M})$

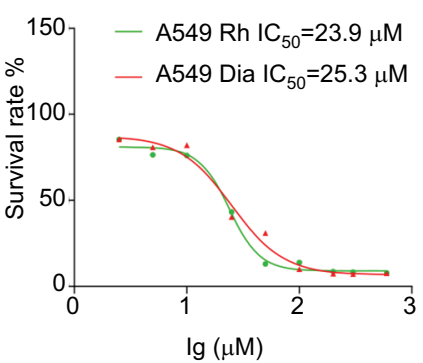

Figure I Rhein inhibits viability and colony formation of NSCLC cells.

Notes: (A) Chemical structure of Rhein and Diacerein. (B) Effects of Rhein and Diacerein inhibit cell viability and colony formation of PC-9. Cells viability: PC-9 cell lines were treated with various concentrations of Rhein and Diacerein for 48 hours, and were analyzed by MTT assay and the IC ${ }_{50}$ values were calculated. Colony formation: PC-9 cells were treated with a concentration gradient of Rhein and Diacerein for 24 hours and incubated for I week; then, the clones were fixed with ice-cold methanol and stained with crystal violet. (C) Effects of Rhein and Diacerein inhibit cell viability and colony formation of H460. (D) Effects of Rhein and Diacerein inhibit cell viability and colony formation of A549.

Abbreviation: NSCLC, non-small-cell lung cancer.

by the MTT assay. The results show that cell viability was suppressed in a dose-dependent manner in the NSCLC cell lines. For comparison, the $\mathrm{IC}_{50}$ values of Rhein and Diacerein were 24.59 and $35.76 \mu \mathrm{M}$ in PC-9 cells (Figure 1B), 52.88 and $63.61 \mu \mathrm{M}$ in $\mathrm{H} 460$ cells (Figure 1C) and $23.9 \mu \mathrm{M}$ and $25.3 \mu \mathrm{M}$ in A549 cells (Figure 1D), respectively. Furthermore, compared with control cells, a dose-dependent decrease in colony formation by PC-9, H460 and A549 cells was detected in colony formation assay. These results suggest that Rhein and Diacerein significantly inhibited the growth and proliferation of human NSCLC cells, inducing cell death in a dose-dependent manner. 


\section{Rhein inhibits the STAT3 signaling pathway}

Based on the important role of STAT3 in the process of NSCLC and the structural similarity of Rhein with the four known inhibitors of STAT3 (Napabucasin, STA-21, LLL12 and LY5), we thought that Rhein may affect the phosphorylation of STAT3. ${ }^{11,21}$ We tested the inhibiting ability of Rhein on STAT3 phosphorylation (P-STAT3) in NSCLC cells. Not unexpectedly, Rhein and Diacerein markedly reduced the P-STAT3 protein level in H460, A549 and PC-9 cell lines.
Rhein and Diacerein decreased P-STAT3 protein expression in NSCLC cells in a concentration-dependent manner after 24 hours of treatment; under the same condition, STAT3 protein expression remained unchanged (Figure $2 \mathrm{~A}-\mathrm{C}$ ). To further confirm the STAT3 inhibitory effect, we also detected the constitutive activation of STAT3 in H460 cells using STAT3 luciferase reporter assay. In agreement with the data obtained using Western blot analysis, we found Rhein significantly blocked P-STAT3 activation in a dose-dependent manner (Figure 2D-E).

A

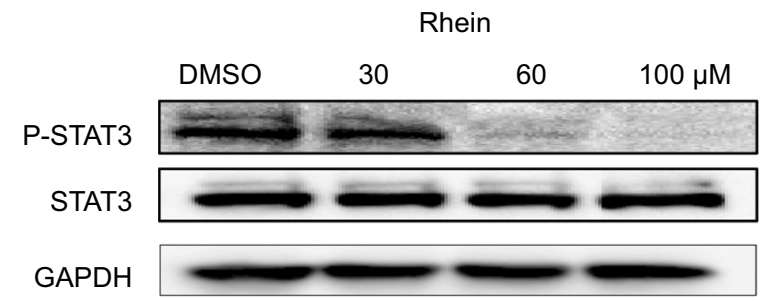

B
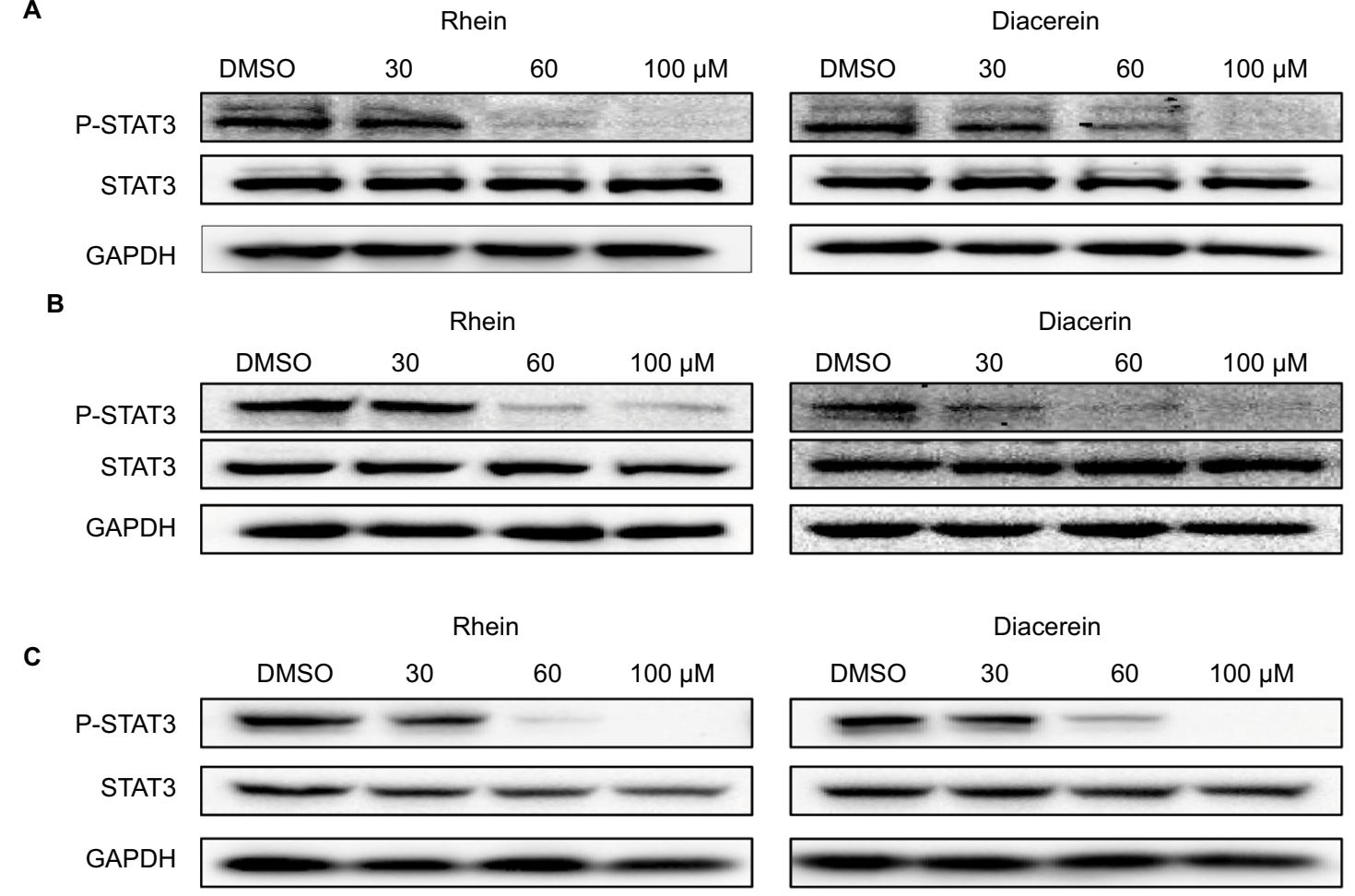

D

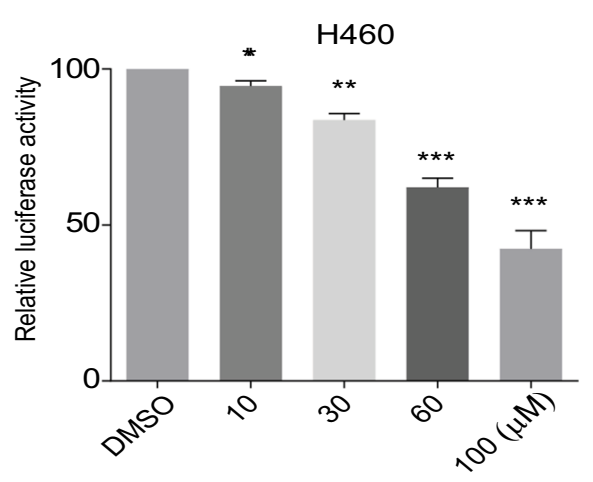

E

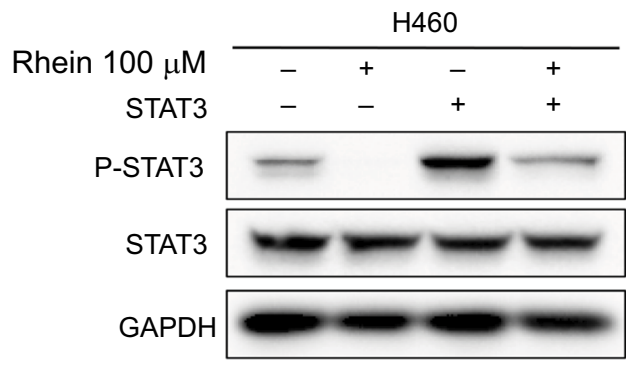

Figure 2 Western blot analysis of P-STAT3 in PC-9, A549 and H460 cells.

Notes: (A) H460 cell lines were treated with a concentration gradient of Rhein and Diacerein. The expression of P-STAT3 was found by Western blot analysis. (B) PC-9 cell lines were treated with a concentration gradient of Rhein and Diacerein. The expression of P-STAT3 was found by Western blot analysis. (C) A549 cell lines were treated with a concentration gradient of Rhein and Diacerein. The expression of P-STAT3 was found by Western blot analysis. (D) H460 cells were transfected with luciferase reporter gene plasmid and treated with Rhein for 24 hours. The results were normalized to the Renilla luciferase activity. The bars indicate the mean \pm SD. Statistically significant differences (Student's $t$-test), ${ }^{* P}<0.05 ; * * P<0.01$; $* * * P<0.00$ I. (E) The STAT3 plasmid was transfected into H460 cells and then treated with Rhein for 24 hours. Cells were then lysed and subjected to immunoblotting with indicated antibodies. GAPDH was used as a loading control. 


\section{Rhein induces apoptosis in human NSCLC cells}

To evaluate the apoptosis-inducing effects of Rhein and Diacerein in H460 and PC-9 cell lines, the cancer cells were treated with Rhein and Diacerein for 48 hours, stained with Annexin V-FITC and PI and the apoptotic cells were evaluated by flow cytometry analysis. The data showed that Rhein and Diacerein dose dependently induced cell apoptosis in PC-9 and H460 cell lines, respectively (Figure 3A-D). In addition, Western blot analysis data further revealed that Rhein and Diacerein increased the expression of the proapoptotic protein Bax and decreased the level of the antiapoptotic protein Bcl-2 in human NSCLC cells in a dose-dependent manner (Figure 3E, F). Our results also revealed that Rhein could regulate the expression of cell death-related proteins, which blocked the process of human NSCLC cell proliferation.

\section{Rhein induces $\mathrm{G} 2 / \mathrm{M}$ cell cycle arrest in human NSCLC cells}

The cell cycle is a process involving a series of cellular events that lead to cell division and ultimately to proliferation. The entire cell cycle can be divided into four stages including the G1 phase, S phase, G2 phase and M phase (the division stage). ${ }^{27}$ To evaluate the cell cycle arrest associated with Rhein, PC-9 and H460 cell lines were treated with Rhein for 24 hours. The percentage of Rheintreated and untreated NSCLC cells in the G2/M phase was measured by flow cytometry analysis. Rhein significantly induced the arrest of NSCLC cells in the G2/M phase of the cell cycle compared with the untreated control group (Figure 4A), following reduced cell growth and increased apoptosis. The Western blot analysis indicated that treatment with Rhein and Diacerein dose dependently inhibited the expression of MDM2, Cyclin B1, CDC2 and P53 in H460 and PC-9 cells (Figure 4B, C). These results indicate that the inhibition of cell proliferation by Rhein is partly associated with the induction of $\mathrm{G} 2 / \mathrm{M}$ phase arrest in $\mathrm{H} 460$ and PC-9 cells.

\section{Rhein inhibits the growth of NSCLC xenograft models}

Given the potent cytotoxic activity of Rhein against human NSCLC cells, we next assessed the therapeutic efficacy of Rhein in H460 xenograft models. Mice were treated with two different doses of Rhein (60 and $100 \mathrm{mg} / \mathrm{kg}$; intra- peritoneally) every other day. Rhein led to a supra-additive reduction in growth in xenograft models (Figure $5 \mathrm{~A}-\mathrm{C}$ ). Mechanistically, we found that STAT3 phosphorylation was inhibited in Rhein treatment groups (Figure 5D). In addition, Rhein treatment markedly increased apoptosis as indicated by the expressions of Bax and the Bcl-2 (Figure 5D). Importantly, the body weight of the mice was stable (Figure 5E). Moreover, Rhein treatment caused no gross toxicities on the heart, liver, kidney and lung compared with the control group (Figure 5F), demonstrating excellent safety profiles.

\section{Discussion}

Recent studies highlight the importance of STAT3 in NSCLC through regulating tumor cell proliferation, survival, tumor invasion and angiogenesis. ${ }^{28,29}$ Several studies have implicated STAT3 activation in EGFR resistance. ${ }^{22,23}$ These suggest that persistent STAT3 may lead to primary resistance to targeted therapies. Thus, the IL-6/JAK/STAT3 signaling pathway seemed to be linked to primary resistance to treatments, including cytotoxic chemotherapy, radiotherapy and targeted therapy. However, up to now, no direct STAT3 inhibitor has been approved for clinical use.

The discovery of small molecular compounds for treating NSCLC is important to improve patient outcome. In this study, we have demonstrated that Rhein inhibited the STAT3 signaling pathway (Figure 2) and thereby induced apoptosis (Figure 3) and reduced the growth of human NSCLC cells in vitro (Figures 1 and 4). More importantly, mice treated with Rhein showed robust inhibition of tumor growth in vivo (Figure 5). Diacerein as a prodrug of Rhein, a known drug for osteoarthritis, also showed the potential for inhibiting IL-6/STAT3 cancer signaling pathway. Consistent with our results, molecular investigations indicated that Diacerein-instigated apoptosis was associated with inhibition of IL-6/STAT3 ${ }^{30,31}$ Natural products with satisfactory clinical efficacy and low toxicity can be used as replacement therapies for many clinical diseases, and such correlative research is one of the hot topics in modern medicine. Notably, we found Rhein had few side effects on the mouse body at our therapeutic concentration (Figure 5E, F). Thus, the discovery of Rhein and Diacerein could speed up the development of clinical therapies for the IL-6/STAT3-dependent cancers.

Collectively, our studies demonstrate that Rhein exhibits the inhibitory potential for NSCLC growth by inhibiting 
A

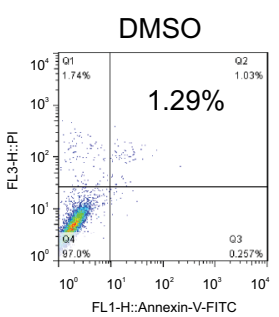

B

DMSO

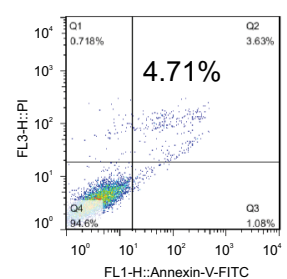

C

DMSO

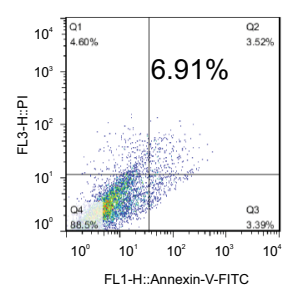

D

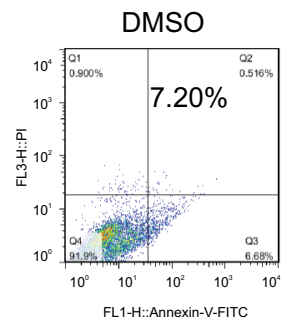

E

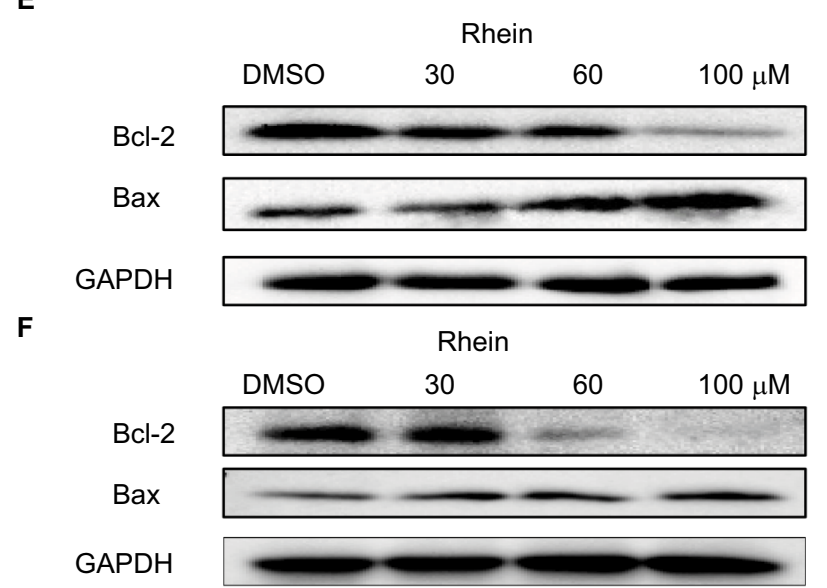

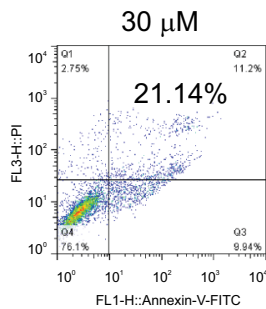

$30 \mu \mathrm{M}$

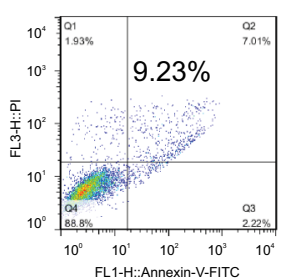

$30 \mu \mathrm{M}$

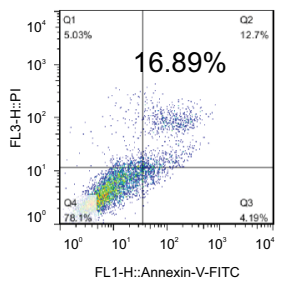

$30 \mu \mathrm{M}$

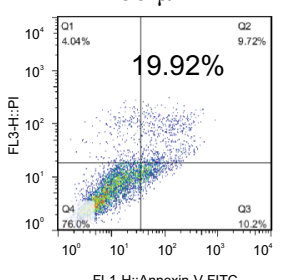

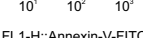

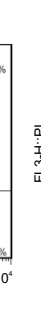

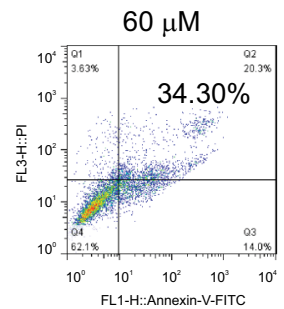

$60 \mu \mathrm{M}$

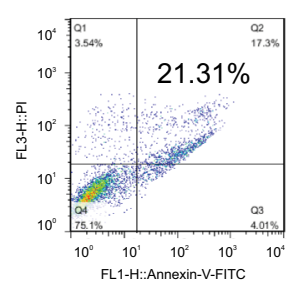

$60 \mu \mathrm{M}$

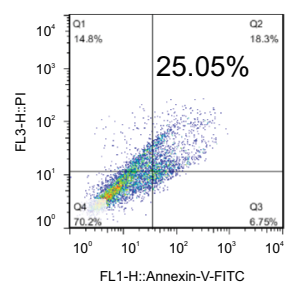

$60 \mu \mathrm{M}$

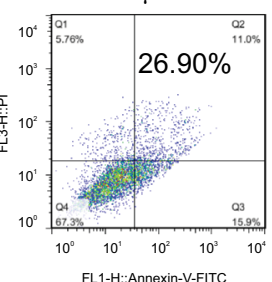

PC-9 Rhein
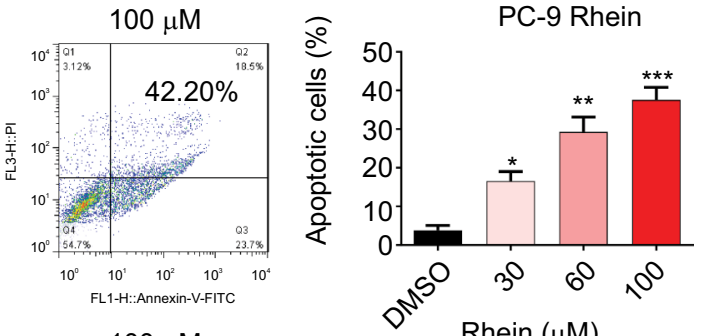

Rhein $(\mu \mathrm{M})$
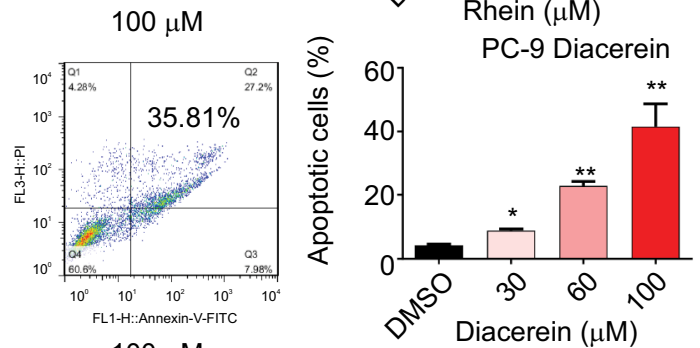

$100 \mu \mathrm{M}$
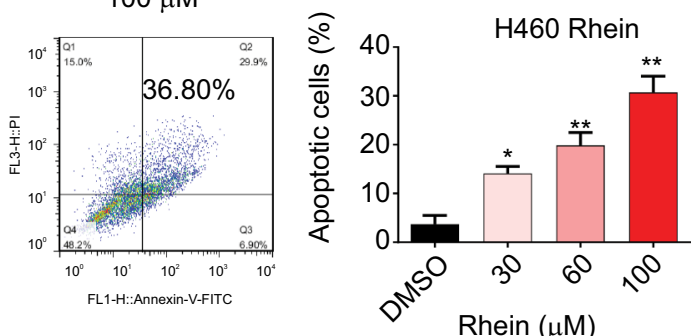

Rhein $(\mu \mathrm{M})$
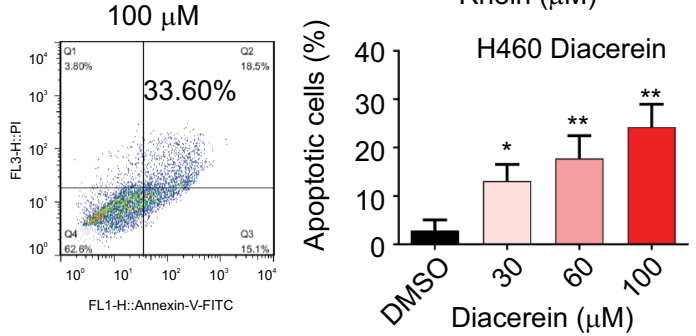

Diaracein

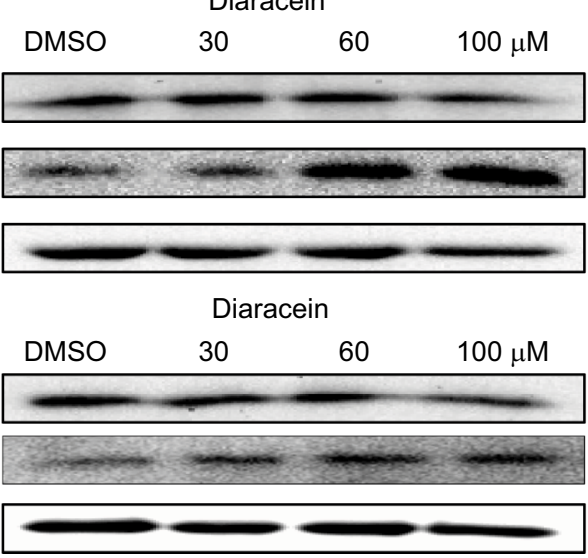

Figure 3 Rhein induced apoptosis of NSCLC cells.

Notes: (A) PC-9 cell lines were treated with a concentration gradient of Rhein for 48 hours and stained with Annexin $V$ and propidium iodide. (B) PC-9 cell lines were treated with a concentration gradient of Diacerein for 48 hours and stained with Annexin $\mathrm{V}$ and propidium iodide. (C) $\mathrm{H} 460$ cell lines were treated with a concentration gradient of Rhein for 48 hours and stained with Annexin $\mathrm{V}$ and propidium iodide. (D) H460 cell lines were treated with a concentration gradient of Diacerein for 48 hours and stained with Annexin $V$ and propidium iodide. Representative histograms from flow cytometry analysis in the two human NSCLC cells treated with various concentrations of Rhein and Diacerein. Assays were performed in triplicate. $* P<0.05$, $* * P<0.01$, and $* * * P<0.001$. (E) Western blot analysis of apoptosis-related proteins. The expression of $\mathrm{Bcl}-2$ and Bax in PC-9 cells. (F) The expression of Bcl-2 and Bax in $\mathrm{H} 460$ cells.

Abbreviations: NSCLC, non-small-cell lung cancer. 
A

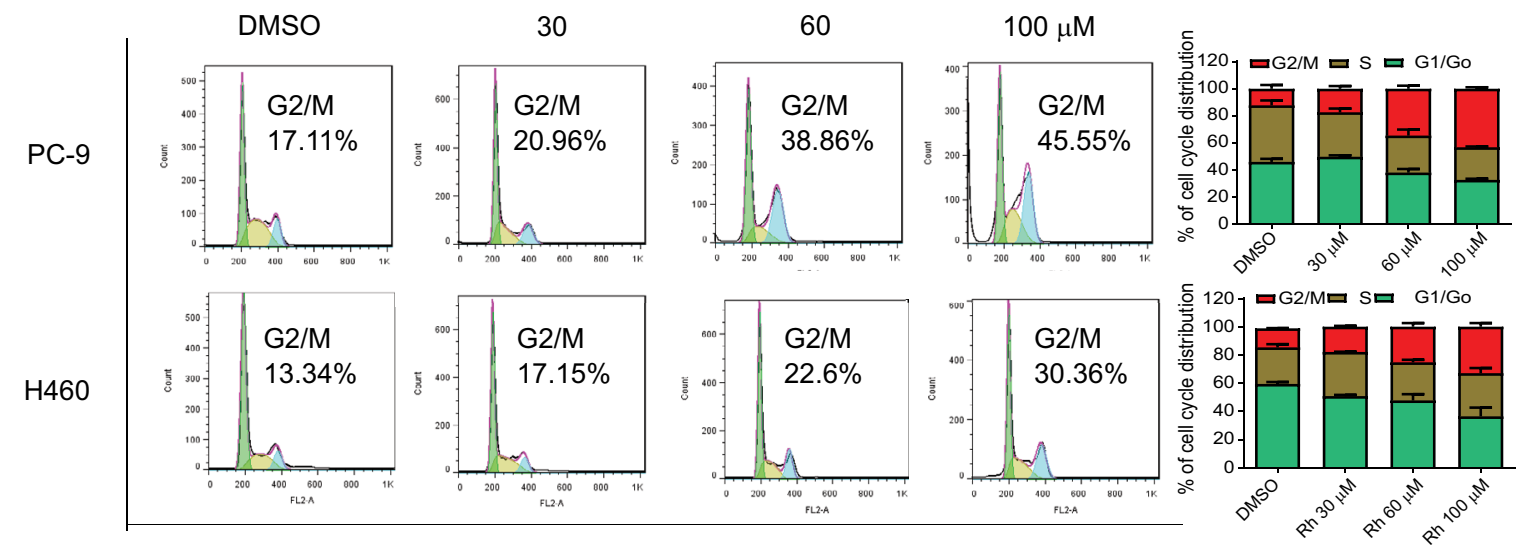

B

Rhein

Diacerein

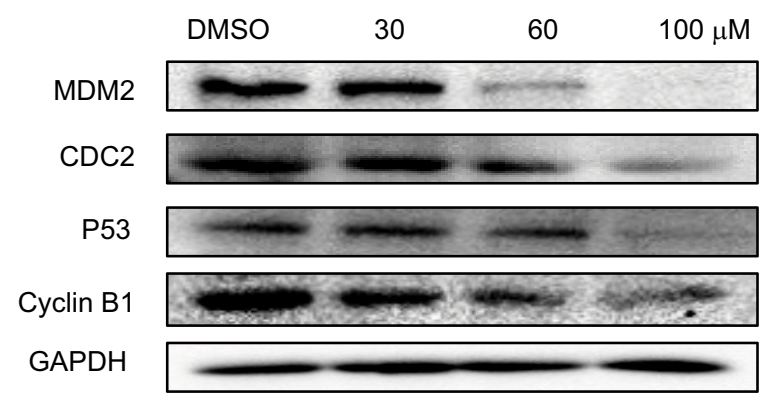

C

Rhein

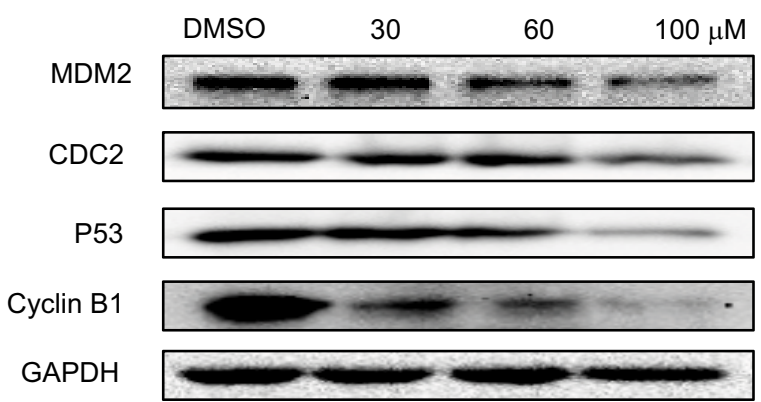

Diacerein

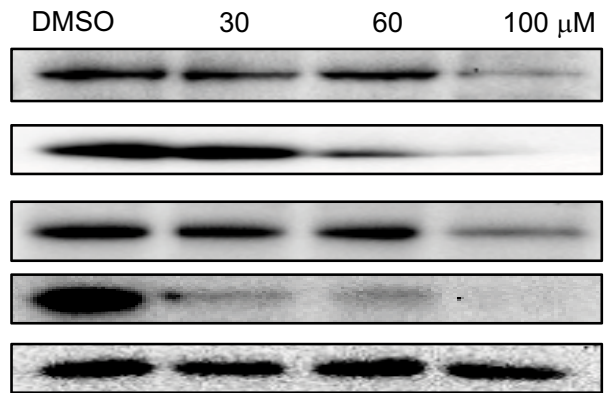

Figure 4 Rhein induces G2/M cell cycle arrest in human NSCLC cell lines.

Notes: (A) Induction of cell cycle arrest in PC-9 and H460 cells analyzed by flow cytometry after treatment with a concentration gradient of Rhein for 24 hours. Representative histograms from flow cytometry analysis in the two human NSCLC cells treated with various concentrations of Rhein. Assays were performed in triplicate. (B) Western blot analysis of cycle-related proteins MDM2, CDC2, Cyclin BI and P53 in PC-9. (C) Western blot analysis of cycle-related proteins MDM2, CDC2, Cyclin BI and P53 in $\mathrm{H} 460$ cells.

Abbreviation: NSCLC, non-small-cell lung cancer.

STAT3 activation. Also, of course, besides potential direct usage for clinic trials, the two compounds can also serve as lead compounds for optimization to speed the development of drugs selectively targeting the IL-6/STAT3 cancer signaling pathway. Additionally, the pharmacokinetics, pharmacodynamics and toxicity of Rhein will be further comprehensively evaluated. We also plan to investigate the combination of Rhein and other clinical cancer drugs to improve the antitumor potency of Rhein and verify its effect on NSCLC.

\section{Acknowledgments}

This work was financially supported by the Medical Scientific Research Fund of Zhejiang Province (2019322308), National Key R\&D Program of China (2017YFA0506000), National Natural Science Foundation of China (81622043), 
A

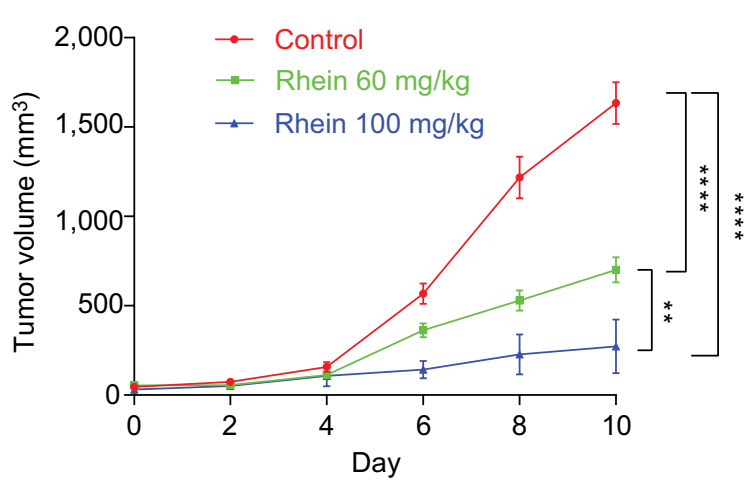

C

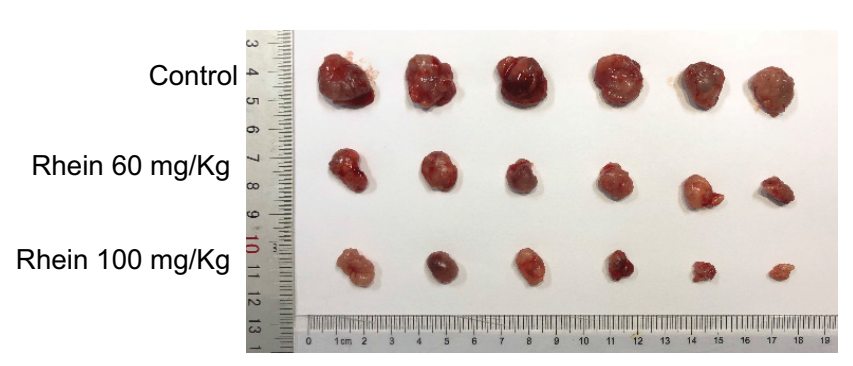

E

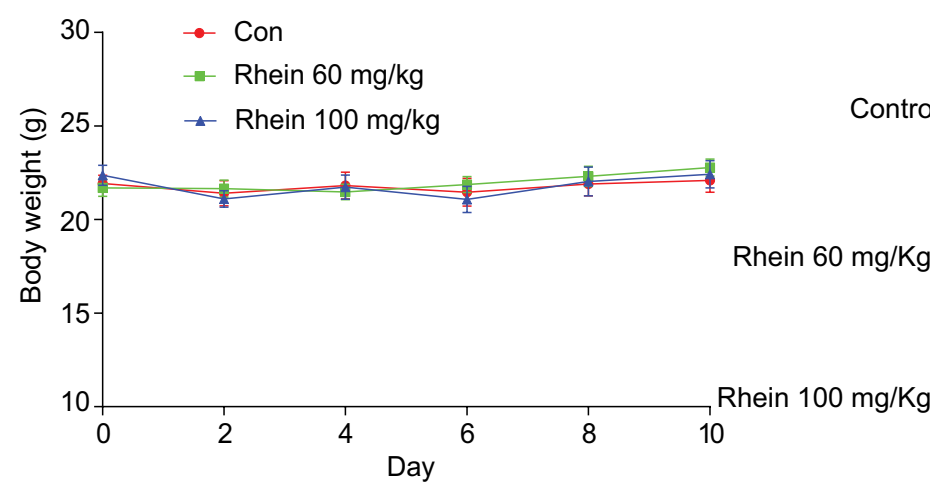

B

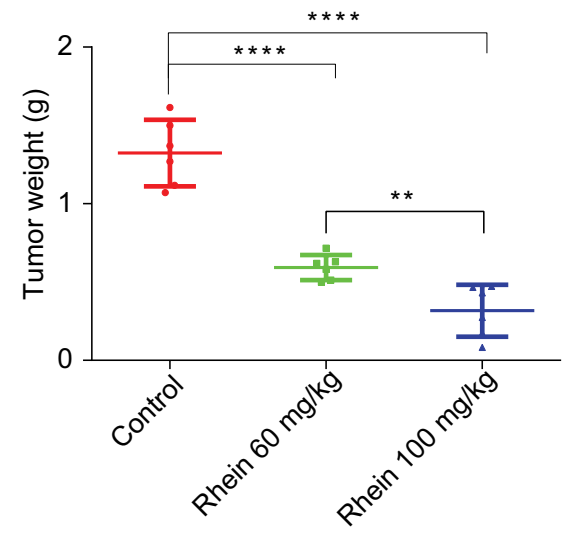

D
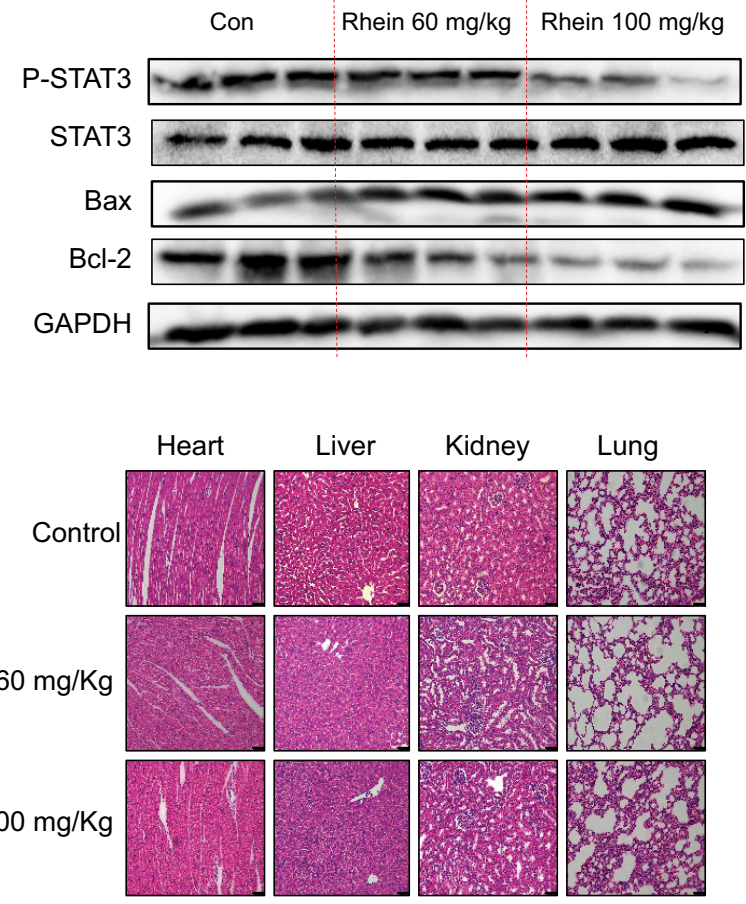

Figure 5 Antitumor activity of Rhein in $\mathrm{H} 460$ xenograft animal model.

Notes: (A) The result of tumor volume. (B) The tumor weight $(n=6)$. (C) Representative images of the tumor tissue in control and treatment groups ( $n=6)$. (D) The tumor tissues were extracted and a Western blot assay was performed. (E) Mice body weight. (F) Kidneys, livers, lungs and hearts tissues from three groups were sectioned at 5 $\mu \mathrm{m}$ and the slides were stained with $\mathrm{H} \& \mathrm{E}$. All images were obtained by microscope with $20 \times$ magnification. $* * P<0.01 * * * * P<0.000 \mathrm{I}$.

Zhejiang Provincial Natural Science Foundation of China (LR16H310001, LY18H160047 and LY17H160055) and Wenzhou Science and Technology Project (Y20170280). We thank Dr Huameng Li for helpful discussions and assistance in writing the manuscript.

\section{Disclosure}

The authors report no conflicts of interest in this work.

\section{References}

1. Ferlay J, Soerjomataram I, Dikshit R, et al. Cancer incidence and mortality worldwide: sources, methods and major patterns in GLOBOCAN 2012. Int J Cancer. 2015;136(5):E359-E386.
2. Yatabe Y, Kerr KM, Utomo A, et al. EGFR mutation testing practices within the Asia Pacific region: results of a multicenter diagnostic survey. J Thorac Oncol. 2015;10(3):438-445.

3. Siegel R, Naishadham D, Jemal A. Cancer statistics, 2012. CA Cancer J Clin. 2012;62(1):10-29.

4. Hirsch FR, Scagliotti GV, Mulshine JL, et al. Lung cancer: current therapies and new targeted treatments. Lancet. 2017;389(10066):299-311.

5. Yu H, Lee H, Herrmann A, Buettner R, Jove R. Revisiting STAT3 signalling in cancer: new and unexpected biological functions. Nat Rev Cancer. 2014;14(11):736-746.

6. Yu H, Pardoll D, Jove R. STATs in cancer inflammation and immunity: a leading role for STAT3. Nat Rev Cancer. 2009;9(11):798-809.

7. Chun J, Li RJ, Cheng MS, Kim YS. Alantolactone selectively suppresses STAT3 activation and exhibits potent anticancer activity in MDA-MB-231 cells. Cancer Lett. 2015;357(1):393-403.

8. Devarajan E, Huang S. STAT3 as a central regulator of tumor metastases. Curr Mol Med. 2009;9(5):626-633. 
9. Yu H, Jove R. The STATs of cancer - new molecular targets come of age. Nat Rev Cancer. 2004;4(2):97-105.

10. Zhang T, Li S, Li J, et al. Natural product pectolinarigenin inhibits osteosarcoma growth and metastasis via SHP-1-mediated STAT3 signaling inhibition. Cell Death Dis. 2016;7(10):e2421.

11. Bai E, Yang L, Xiang Y, et al. L61H46 shows potent efficacy against human pancreatic cancer through inhibiting STAT3 pathway. Cancer Manag Res. 2018;10:565-581.

12. Zheng H, Hong H, Zhang L, et al. Nifuratel, a novel STAT3 inhibitor with potent activity against human gastric cancer cells. Cancer Manag Res. 2017;9:565-572.

13. Cortas T, Eisenberg R, Fu P, Kern J, Patrick L, Dowlati A. Activation state EGFR and STAT-3 as prognostic markers in resected non-small cell lung cancer. Lung Cancer. 2007;55(3):349-355.

14. Gao SP, Mark KG, Leslie K, et al. Mutations in the EGFR kinase domain mediate STAT3 activation via IL-6 production in human lung adenocarcinomas. J Clin Invest. 2007;117(12):3846-3856.

15. Haura EB, Zheng Z, Song L, Cantor A, Bepler G. Activated epidermal growth factor receptor-Stat-3 signaling promotes tumor survival in vivo in non-small cell lung cancer. Clin Cancer Res. 2005;11(23):8288-8294.

16. Barré B, Vigneron A, Perkins N, Roninson IB, Gamelin E, Coqueret O. The STAT3 oncogene as a predictive marker of drug resistance. Trends Mol Med. 2007;13(1):4-11.

17. Ikuta K, Takemura K, Kihara M, et al. Overexpression of constitutive signal transducer and activator of transcription $3 \mathrm{mRNA}$ in cisplatin-resistant human non-small cell lung cancer cells. Oncol Rep. 2005;13(2):217-222.

18. You S, Li R, Park D, et al. Disruption of STAT3 by niclosamide reverses radioresistance of human lung cancer. Mol Cancer Ther. 2014;13(3):606-616.

19. Zhong Z, Wen Z, Darnell JE Jr. STAT3: a STAT family member activated by tyrosine phosphorylation in response to epidermal growth factor and interleukin-6. Science. 1994;264(5155):95-98.

20. Alvarez JV, Greulich H, Sellers WR, Meyerson M, Frank DA. Signal transducer and activator of transcription 3 is required for the oncogenic effects of non-small-cell lung cancer-associated mutations of the epidermal growth factor receptor. Cancer Res. 2006;66(6):3162-3168.
21. Zhao C, Li H, Lin HJ, Yang S, Lin J, Liang G. Feedback activation of STAT3 as a cancer drug-resistance mechanism. Trends Pharmacol Sci. 2016;37(1):47-61.

22. Lee HJ, Zhuang G, Cao Y, Du P, Kim HJ, Settleman J. Drug resistance via feedback activation of STAT3 in oncogene-addicted cancer cells. Cancer Cell. 2014;26(2):207-221.

23. Van Schaeybroeck S, Kalimutho M, Dunne PD, et al. ADAM17-dependent c-MET-STAT3 signaling mediates resistance to MEK inhibitors in KRAS mutant colorectal cancer. Cell Rep. 2014;7(6):1940-1955.

24. Costa DB, Nguyen KS, Cho BC, et al. Effects of erlotinib in EGFR mutated non-small cell lung cancers with resistance to gefitinib. Clin Cancer Res. 2008;14(21):7060-7067.

25. Zhou YX, Xia W, Yue W, Peng C, Rahman K, Zhang H. Rhein: a review of pharmacological activities. Evid Based Complement Alternat Med. 2015;2015(1):1-10

26. Debord P, Louchahi K, Tod M, Cournot A, Perret G, Petitjean O. Influence of renal function on the pharmacokinetics of diacerein after a single oral dose. Eur J Drug Metab Pharmacokinet. 1994;19(1):13-19.

27. Jang W, Kim T, Koo JS, Kim SK, Lim DS. Mechanical cue-induced YAP instructs Skp2-dependent cell cycle exit and oncogenic signaling. EMBO J. 2017;36(17):2510-2528.

28. Jiang R, Jin Z, Liu Z, Sun L, Wang L, Li K. Correlation of activated STAT3 expression with clinicopathologic features in lung adenocarcinoma and squamous cell carcinoma. Mol Diagn Ther. 2011;15(6): 347-352.

29. XuYH, Lu SX. A meta-analysis of STAT3 and phospho-STAT3 expression and survival of patients with non-small-cell lung cancer. Eur J Surg Oncol. 2014;40(3):311-317.

30. Bharti R, Dey G, Banerjee I, et al. Somatostatin receptor targeted liposomes with Diacerein inhibit IL-6 for breast cancer therapy. Cancer Lett. 2017;388:292-302.

31. Bharti R, Dey G, Ojha PK, et al. Diacerein-mediated inhibition of IL-6/ IL-6R signaling induces apoptotic effects on breast cancer. Oncogene. 2016;35(30):3965-3975.
Cancer Management and Research

\section{Publish your work in this journal}

Cancer Management and Research is an international, peer-reviewed open access journal focusing on cancer research and the optimal use of preventative and integrated treatment interventions to achieve improved outcomes, enhanced survival and quality of life for the cancer patient. The manuscript management system is completely online and includes

\section{Dovepress}

a very quick and fair peer-review system, which is all easy to use. Visit $\mathrm{http}: / / \mathrm{www}$.dovepress.com/testimonials.php to read real quotes from published authors. 